the adapted therapy, but we did adapt the devised therapy, and superficial scrutiny of the authors on both the papers cited will attest to this. We stand by our conclusion that the model can be adapted and that, at least in part, it works. This is more than anyone else has ever done in this field, and one could only agree with Dr White's comment about future research in this area.

A. Burns Department of Psychiatry, 2nd Floor, Education and Research Centre,Wythenshawe Hospital, Manchester M23 9LT, UK.

E-mail: alistair.burns@manchester.ac.uk

\section{Call for a European Guidelines Institute}

The guideline assessment by Gaebel et al (2005) has been long overdue. Whereas there was originally quite a bit of scepticism about guidelines in psychiatry, there now appears to be a sort of 'guidelines mania', as each European national association tries to produce its own guidelines. We have recently completed an assessment of even more $(n=61)$ guidelines, which was not limited to schizophrenia but focused on European psychiatric guidelines. Although our results were similar - the general quality of the guidelines was medium grade, although there were some of outstanding quality - we arrived at somewhat different conclusions.

By amending the AGREE instrument (AGREE Collaboration, 2003) with an additional item, we found that national particularities were very rarely considered by European psychiatric guidelines (18\%), and then only very vaguely. This is not surprising, since the evidence available for guidelines is almost always of an international nature. There are hardly any treatment studies focusing specifically on national subgroups. Therefore the evidence underlying the guidelines must always be the same per se. Given the enormous expenditure of time and money that is necessary to develop a methodologically sound guideline, we strongly advocate the establishment of a European Guidelines Institute. It could then be the task of the national associations to adapt guidelines to the specific conditions of their own countries.
Such a procedure could improve the quality of most national guidelines and foster the ongoing standardisation of European medical care.

AGREE Collaboration (2003) Development and validation of an international guidelines appraisal instrument for assessing the quality of clinical practice guidelines: the AGREE project. Quality and Safety in Health Care, 12, 18-23.

Gaebel, W., Weinmann, S., Sartorius, N., et al (2005) Schizophrenia practice guidelines: international survey and comparison. British Journal of Psychiatry, $\mathbf{1 8 7}$, 248-255.

S. Leucht Department of Psychiatry and Psychotherapy, Technische Universität München, Ismaningerstrasse 22, 81675 Munich, Germany. E-mail: Stefan.Leucht@|rz.tum.de

M. Stiegler, C. Rummel, K. Wahlbeck, W. Kissling Department of Psychiatry and Psychotherapy, Technische Universität München, Munich, Germany

Authors' reply: Leucht $e t$ al agree with us that specific national conditions are rarely considered by practice guidelines. This is not surprising because the evidence available for guidelines is almost always of an international nature. However, in our view not all guidelines share or should share the same evidence. Those developing guidelines may ask different clinical questions and may consider different interventions or outcomes as relevant. This is not a methodological problem of guidelines.

Furthermore, methodologically sound qualitative evidence such as consumer preference studies may be used by guideline developers; this may be restricted to certain regions or nations. It is certainly of concern, however, that the external validity of the available study evidence is rarely evaluated. There are also few efforts to analyse minority ethnic or other subgroups within multicentre studies or to run effectiveness studies in nonWestern countries. Of the 5000 randomised controlled trials (RCTs) in the database of the Cochrane Schizophrenia Group, 80\% are from Western countries (Moll et al, 2003). The American Psychiatric Association's 1997 clinical practice guideline for schizophrenia does not provide any information regarding potentially different outcomes for minority ethnic groups included in the RCTs from the USA (U.S. Department of Health \& Human Services, 2001).

It is not obvious to us that a European Guidelines Institute would be of great help or would be accepted as a legitimate developer of guidelines. During the development of guidelines many decisions must be made at an early stage by national consensus groups with the contribution of key stakeholders. In our survey most respondents preferred to develop national guidelines with the help of international experts or to share experiences or data with other guideline developers. In our view, evidence concerning efficacy and risk of specific interventions could be reviewed by an international group in order to develop core recommendations that could be adapted by those developing national or local guidelines. For this purpose activities have been started within the World Psychiatric Association (WPA). However, the adaptation of available evidence to local circumstances is an important national or regional duty in guideline development.

\section{Declaration of interest}

W.G. is Chairman of the Section on Schizophrenia of the WPA and Chairman of the Section on Guideline Development of the German Society of Psychiatry, Psychotherapy and Nervous Diseases (DGPPN). S.W. is involved in the revision of the schizophrenia practice guideline of the DGPPN.

Moll, C., Gessler, U., Bartsch, S., et al (2003) Gross domestic product and productivity of schizophrenia trials: an ecological study. BMC Psychiatry, 3, I-5.

U.S. Department of Health \& Human Services (200I) Mental Health: Culture, Race, Ethnicity - A Supplement to Mental Health: A Report of the Surgeon General. Rockville, MD: US Department of Health and Human Services. http: //

www. mentalhealth.samhsa.gov/cre/default.asp

W. Gaebel Department of Psychiatry and Psychotherapy, Heinrich Heine University

Düsseldorf, Bergische Landstrasse 2, D-40629

Düsseldorf, Germany.

E-mail:Wolfgang.Gaebel@uni-duesseldorf.de

S. Weinmann Department of Psychiatry II, University of Ulm, Germany 